\title{
Publisher Correction: Raising Girls with Emotional and Behavioral Challenges: An Exploration of Caregiver Perceptions
}

\author{
Elisabeth H. Rice ${ }^{1} \cdot$ Margaux H. Brown $\mathbb{1}^{2}$ - Darcie Whitlow ${ }^{3} \cdot$ Karen Ihrig ${ }^{1} \cdot$ Kandace M. Hoppin ${ }^{4} \cdot$ Melissa Boston $^{1}$. \\ Adelaide Kelly-Massoud ${ }^{1} \cdot$ Amy Srsic $^{5}$
}

Published online: 18 March 2020

(c) Springer Science+Business Media, LLC, part of Springer Nature 2020

\section{Correction to: Journal of Child and Family Studies} https://doi.org/10.1007/s10826-020-01702-8

The original version of this article unfortunately contained a mistake.

The error pertains to Discussion, Paragraph 2, beginning with the second sentence. After the first sentence of the paragraph, the rest of the paragraph should read:

These multifaceted behavioral profiles suggest that professionals should look beyond the stereotypical notion of "sad girls" and "bad boys" (Peter and Roberts 2010). Future researchers should also consider if measuring the behaviors for girls with emotional and behavioral challenges using an externalizing and internalizing continuum is an accurate way to measure their behaviors. This finding echoes conclusions from Vaughan et al. (2012), who noted that a child's expression of both externalizing and internalizing behaviors is linked to greater caregiver strain.

The original article has been corrected.

The original article can be found online at https://doi.org/10.1007/ s10826-020-01702-8.

Margaux H. Brown

mbrown4@augusta.edu

1 Department of Special Education and Disability Studies, The George Washington University, Washington, DC, USA

2 Department of Advanced Studies and Innovation, Augusta University, Augusta, GA, USA

3 Great Prairie Area Education Agency, Ottumwa, IA, USA

4 Department of Special Education, Towson University, Towson, MD, USA

5 Instruction and Learning, University of Pittsburgh, Pittsburgh, PA, USA 